\title{
A Class of Nonlinear Integral Operators Preserving Double Subordinations
}

\author{
Oh Sang Kwon ${ }^{1}$ and Nak Eun Cho ${ }^{2}$ \\ ${ }^{1}$ Department of Mathematics, Kyungsung University, Pusan 608-736, South Korea \\ ${ }^{2}$ Department of Applied Mathematics, Pukyong National University, Pusan 608-737, South Korea \\ Correspondence should be addressed to Nak Eun Cho, necho@pknu.ac.kr \\ Received 15 February 2008; Accepted 8 May 2008 \\ Recommended by Stevo Stevic
}

The purpose of the present paper is to investigate some subordination- and superordinationpreserving properties of certain integral operators defined on the space of meromorphic functions in the punctured open unit disk. The sandwich-type theorem for these integral operators is also considered.

Copyright ( 12008 O. S. Kwon and N. E. Cho. This is an open access article distributed under the Creative Commons Attribution License, which permits unrestricted use, distribution, and reproduction in any medium, provided the original work is properly cited.

\section{Introduction}

Let $\mathscr{H}=\mathscr{H}(\mathbb{U})$ denote the class of analytic functions in the open unit disk $\mathbb{U}=\{z \in \mathbb{C}:|z|<1\}$. For $a \in \mathbb{C}$, let

$$
\mathscr{L}[a, n]=\left\{f \in \mathscr{H}: f(z)=a+a_{n} z^{n}+a_{n+1} z^{n+1}+\cdots\right\} .
$$

Let $f$ and $F$ be members of $\mathscr{\ell}$. The function $f$ is said to be subordinate to $F$, or $F$ is said to be superordinate to $f$, if there exists a function $w$ analytic in $\mathbb{U}$, with $w(0)=0$ and $|w(z)|<1$, and such that $f(z)=F(w(z))$. In such a case, we write $f<F$ or $f(z)<F(z)$. If the function $F$ is univalent in $\mathbb{U}$, then we have $f \prec F$ if and only if $f(0)=F(0)$ and $f(\mathbb{U}) \subset F(\mathbb{U})$ (cf. [1]).

Definition 1.1 (see [1]). Let $\phi: \mathbb{C}^{2} \rightarrow \mathbb{C}$ and let $h$ be univalent in $\mathbb{U}$. If $p$ is analytic in $\mathbb{U}$ and satisfies the differential subordination

$$
\phi\left(p(z), z p^{\prime}(z)\right) \prec h(z),
$$

then $p$ is called a solution of the differential subordination. The univalent function $q$ is called a dominant of the solutions of the differential subordination, or more simply a dominant if $p<q$ 
for all $p$ satisfying (1.2). A dominant $\tilde{q}$ that satisfies $\tilde{q} \prec q$ for all dominants $q$ of (1.2) is said to be the best dominant.

Definition 1.2 (see [2]). Let $\varphi: \mathbb{C}^{2} \rightarrow \mathbb{C}$ and let $h$ be analytic in $\mathbb{U}$. If $p$ and $\varphi\left(p(z), z p^{\prime}(z)\right)$ are univalent in $\mathbb{U}$ and satisfy the differential superordination

$$
h(z) \prec \varphi\left(p(z), z p^{\prime}(z)\right),
$$

then $p$ is called a solution of the differential superordination. An analytic function $q$ is called a subordinant of the solutions of the differential superordination, or more simply a subordinant if $q \prec p$ for all $p$ satisfying (1.3). A univalent subordinant $\tilde{q}$ that satisfies $q \prec \tilde{q}$ for all subordinants $q$ of (1.3) is said to be the best subordinant.

Definition 1.3 (see [2]). One denotes by $Q$ the class of functions $f$ that are analytic and injectiveon $\overline{\mathbb{U}} \backslash E(f)$, where

$$
E(f)=\left\{\zeta \in \partial \mathbb{U}: \lim _{z \rightarrow \zeta} f(z)=\infty\right\},
$$

and are such that $f^{\prime}(\zeta) \neq 0$ for $\zeta \in \partial \mathbb{U} \backslash E(f)$.

Let $\Sigma$ denote the class of functions of the form

$$
f(z)=\frac{1}{z}+\sum_{n=0}^{\infty} a_{n} z^{n}
$$

which are analytic in the punctured open unit disk $\mathbb{D}=\mathbb{U} \backslash\{0\}$. Let $\Sigma^{*}$ and $\Sigma_{k}$ be the subclasses of $\Sigma$ consisting of all functions which are, respectively, meromorphic starlike and meromorphic convex in $\mathbb{D}$ (see, for details, [3-5]).

For a function $f \in \Sigma$, we introduce the following integral operators $I_{\beta, \gamma}$ defined by

$$
I_{\beta, \gamma}(f)(z):=\left(\frac{\gamma-\beta+1}{z^{\gamma+1}} \int_{0}^{z} t^{\gamma} f^{\beta}(t) d t\right)^{1 / \beta} \quad(f \in \Sigma ; \beta, \gamma \in \mathbb{C} ; \beta \in \mathbb{C} \backslash\{0\} ; \operatorname{Re}\{\gamma-\beta\}>0) \text {. }
$$

The integral operator $I_{\beta, \gamma}(f)$ defined by (1.6) has been extensively studied by many authors [6-10] with suitable restrictions on the parameters $\beta$ and $\gamma$, and for $f$ belonging to some favored classes of meromorphic functions. In particular, Bajpai [6] showed that the integral operator $I_{1,1}(f)$ belongs to the classes $\Sigma^{*}$ and $\Sigma_{k}$, whenever $f$ belongs to the classes $\Sigma^{*}$ and $\Sigma_{k}$, respectively. Moreover, the operator $I_{\beta, \gamma}$ for the case $\beta=1$ is related to the generalized Libera transform introduced by Stević (see, e.g., [11-13]).

Making use of the principle of subordination between analytic functions, Miller et al. [14] obtained some subordination-preserving properties for certain integral operators (see also [15]). Moreover, Miller and Mocanu [2] considered differential superordinations as the dual concept of differential subordinations (see also [16]). In the present paper, we obtain the subordination- and superordination-preserving properties of the integral operator $I_{\beta, \gamma}$ defined by (1.6) with the sandwich-type theorem.

Throughout this paper, we denote the class $\Sigma_{\beta, \gamma}$ by

$$
\Sigma_{\beta, \gamma}:=\left\{f \in \Sigma: z f(z) \neq 0 \text { and } z I_{\beta, r}(f)(z) \neq 0(z \in \mathbb{U} ; \beta \neq 1)\right\},
$$


where $I_{\beta, \gamma}$ is the integral operator defined by (1.6). For various interesting developments involving functions in the class $\Sigma_{\beta, \gamma}$, the reader may be referred, for example, to the work of Dwivedi et al. [9].

\section{A set of lemmas}

The following lemmas will be required in our present investigation.

Lemma 2.1 (see [17]). Suppose that the function $H: \mathbb{C}^{2} \rightarrow \mathbb{C}$ satisfies the following condition:

$$
\operatorname{Re}\{H(i s, t)\} \leq 0
$$

for all real $s$ and $t \geq-n\left(1+s^{2}\right) / 2$, where $n$ is a positive integer. If the function $p(z)=1+p_{n} z^{n}+\cdots$ is analytic in $\mathbb{U}$ and

$$
\operatorname{Re}\left\{H\left(p(z), z p^{\prime}(z)\right)\right\}>0 \quad(z \in \mathbb{U}),
$$

then $\operatorname{Re}\{p(z)\}>0(z \in \mathbb{U})$.

Lemma 2.2 (see [5]). Let $\beta, \gamma \in \mathbb{C}$ with $\beta \neq 0$ and let $h \in \mathscr{\ell}(\mathbb{U})$ with $h(0)=c$. If $\operatorname{Re}\{\beta h(z)+\gamma\}>$ $0(z \in \mathbb{U})$, then the solution of the differential equation

$$
q(z)+\frac{z q^{\prime}(z)}{\beta q(z)+\gamma}=h(z) \quad(z \in \mathbb{U} ; q(0)=c)
$$

is analytic in $\mathbb{U}$ and satisfies $\operatorname{Re}\{\beta q(z)+\gamma\}>0(z \in \mathbb{U})$.

Lemma 2.3 (see [1]). Let $p \in \mathcal{Q}$ with $p(0)=a$ and let $q(z)=a+a_{n} z^{n}+\cdots$ be analytic in $\mathbb{U}$ with $q(z) \not \equiv a$ and $n \geq 1$. If $q$ is not subordinate to $p$, then there exist points $z_{0}=r_{0} \mathrm{e}^{i \theta} \in \mathbb{U}$ and $\zeta_{0} \in \partial \mathbb{U} \backslash E(f)$, for which

$$
q\left(\mathbb{U}_{r_{0}}\right) \subset p(\mathbb{U}), \quad q\left(z_{0}\right)=p\left(\zeta_{0}\right), \quad z_{0} q^{\prime}\left(z_{0}\right)=m \zeta_{0} p^{\prime}\left(\zeta_{0}\right) \quad(m \geq n) .
$$

A function $L(z, t)$ defined on $\mathbb{U} \times[0, \infty)$ is the subordination chain (or Löwner chain) if $L(\cdot, t)$ is analytic and univalent in $\mathbb{U}$ for all $t \in[0, \infty), L(z, \cdot)$ is continuously differentiable on $[0, \infty)$ for all $z \in \mathbb{U}$, and $L(z, s) \prec L(z, t)$ for $0 \leq s<t$.

Lemma 2.4 (see [2]). Let $q \in \mathscr{H}[a, 1]$, set $\varphi: \mathbb{C}^{2} \rightarrow \mathbb{C}$, and let $\varphi\left(q(z), z q^{\prime}(z)\right) \equiv h(z)(z \in \mathbb{U})$. If $L(z, t)=\varphi\left(q(z), t z q^{\prime}(z)\right)$ is a subordination chain and $p \in \mathscr{L}[a, 1] \cap \mathcal{Q}$, then

$$
h(z) \prec \varphi\left(p(z), z p^{\prime}(z)\right)
$$

implies that

$$
q(z) \prec p(z) .
$$

Furthermore, if $\varphi\left(q(z), z p^{\prime}(z)\right)=h(z)$ has a univalent solution $q \in \mathcal{Q}$, then $q$ is the best subordinant.

Lemma 2.5 (see [18]). The function $L(z, t)=a_{1}(t) z+\cdots$ with $a_{1}(t) \neq 0$ and $\lim _{t \rightarrow \infty}\left|a_{1}(t)\right|=\infty$ is a subordination chain if and only if

$$
\operatorname{Re}\left\{\frac{z \partial L(z, t) / \partial z}{\partial L(z, t) / \partial t}\right\}>0 \quad(z \in \mathbb{U} ; 0 \leq t<\infty) .
$$




\section{Main results}

Subordination theorem involving the integral operator $I_{\beta, \gamma}$ defined by (1.6) is contained in Theorem 3.1 below.

Theorem 3.1. Let $f, g \in \Sigma_{\beta, \gamma}$. Suppose that

$$
\operatorname{Re}\left\{1+\frac{z \phi^{\prime \prime}(z)}{\phi^{\prime}(z)}\right\}>-\delta \quad\left(z \in \mathbb{U} ; \phi(z):=z(z g(z))^{\beta}\right)
$$

where

$$
\delta=\frac{1+|\gamma-\beta|^{2}-\left|1-(\gamma-\beta)^{2}\right|}{4 \operatorname{Re}\{\gamma-\beta\}} \quad(\operatorname{Re}\{\gamma-\beta\}>0) .
$$

Then, the subordination

$$
z(z f(z))^{\beta} \prec z(z g(z))^{\beta}
$$

implies that

$$
z\left(z I_{\beta, \gamma}(f)(z)\right)^{\beta}<z\left(z I_{\beta, \gamma}(g)(z)\right)^{\beta}
$$

where $I_{\beta, r}$ is the integral operator defined by (1.6). Moreover, the function $z\left(z I_{\beta, \gamma}(g)(z)\right)^{\beta}$ is the best dominant.

Proof. Let us define the functions $F$ and $G$ by

$$
F(z):=z\left(z I_{\beta, \gamma}(f)(z)\right)^{\beta}, \quad G(z):=z\left(z I_{\beta, \gamma}(g)(z)\right)^{\beta},
$$

respectively. Without loss of generality, we can assume that $G$ is analytic and univalent on $\overline{\mathbb{U}}$ and that

$$
G^{\prime}(\zeta) \neq 0 \quad(|\zeta|=1)
$$

We first show that if the function $q$ is defined by

$$
q(z):=1+\frac{z G^{\prime \prime}(z)}{G^{\prime}(z)} \quad(z \in \mathbb{U})
$$

then

$$
\operatorname{Re}\{q(z)\}>0 \quad(z \in \mathbb{U}) .
$$

In terms of the function $\phi$ involved in (3.1), the definition (1.6) readily yields

$$
\beta \frac{z\left(I_{\beta, \gamma}(g)(z)\right)^{\prime}}{I_{\beta, \gamma}(g)(z)}=-(\gamma+1)+(\gamma-\beta+1) \frac{\phi(z)}{G(z)} .
$$


We also have

$$
\beta \frac{z\left(I_{\beta, \gamma}(g)(z)\right)^{\prime}}{I_{\beta, \gamma}(g)(z)}=-(1+\beta)+\frac{z G^{\prime}(z)}{G(z)} .
$$

By a simple calculation with (3.9) and (3.10), we obtain the relationship

$$
1+\frac{z \phi^{\prime \prime}(z)}{\phi^{\prime}(z)}=q(z)+\frac{z q^{\prime}(z)}{q(z)+\gamma-\beta} \equiv h(z) .
$$

From (3.1), we note that

$$
\operatorname{Re}\{h(z)+\gamma-\beta\}>0 \quad(z \in \mathbb{U})
$$

and by using Lemma 2.2, we conclude that the differential equation (3.11) has a solution $q \in$ $\mathscr{H}(\mathbb{U})$ with

$$
q(0)=h(0)=1
$$

Let us put

$$
H(u, v)=u+\frac{v}{u+\gamma-\beta}+\delta
$$

where $\delta$ is given by (3.2). From (3.1), (3.11), and (3.14), we obtain

$$
\operatorname{Re}\left\{H\left(q(z), z q^{\prime}(z)\right)\right\}>0 \quad(z \in \mathbb{U}) .
$$

Now, we proceed to show that $\operatorname{Re}\{H(i s, t)\} \leq 0$ for all real $s$ and $t \leq-\left(1+s^{2}\right) / 2$. From (3.14), we have

$$
\operatorname{Re}\{H(i s, t)\}=\operatorname{Re}\left\{i s+\frac{t}{i s+\gamma-\beta}+\delta\right\}=\frac{t \operatorname{Re}\{\gamma-\beta\}}{|\gamma-\beta+i s|^{2}}+\delta \leq-\frac{E_{\delta}(s)}{2|\gamma-\beta+i s|^{2}},
$$

where

$$
E_{\delta}(s):=(\operatorname{Re}\{\gamma-\beta\}-2 \delta) s^{2}-4 \delta \operatorname{Im}\{\gamma-\beta\} s-2 \delta|\gamma-\beta|^{2}+\operatorname{Re}\{\gamma-\beta\} \text {. }
$$

For $\delta$ given by (3.2), we note that the coefficient of $s^{2}$ in the quadratic expression $E_{\delta}(s)$ given by (3.17) is positive or equal to zero. Moreover, for the assumed value of $\delta$ given by (3.2), the quadratic expression $E_{\delta}(s)$ by $s$ in (3.17) is a perfect square. Hence, from (3.16), we see that $\operatorname{Re}\{H(i s, t)\} \leq 0$ for all real $s$ and $t \leq-\left(1+s^{2}\right) / 2$. Thus, by using Lemma 2.1, we conclude that

$$
\operatorname{Re}\{q(z)\}>0 \quad(z \in \mathbb{U}),
$$

that is, $G$ is convex in $\mathbb{U}$.

Next, we prove that the subordination condition (3.3) implies that

$$
F(z) \prec G(z)
$$


for the functions $F$ and $G$ defined by (3.5). For this purpose, we consider the function $L(z, t)$ given by

$$
L(z, t):=\frac{\gamma-\beta}{\gamma-\beta+1} G(z)+\frac{1+t}{\gamma-\beta+1} z G^{\prime}(z) \quad(z \in \mathbb{U} ; 0 \leq t<\infty)
$$

Since $G$ is convex in $\mathbb{U}$ and $\operatorname{Re}\{\gamma-\beta\}>0$, we obtain that

$$
\begin{aligned}
&\left.\frac{\partial L(z, t)}{\partial z}\right|_{z_{0}}=G^{\prime}(0)\left(1+\frac{t}{\gamma-\beta+1}\right) \neq 0 \quad(z \in \mathbb{U} ; 0 \leq t<\infty), \\
& \operatorname{Re}\left\{\frac{z \partial L(z, t) / \partial z}{\partial L(z, t) / \partial t}\right\}=\operatorname{Re}\left\{\gamma-\beta+(1+t)\left(1+\frac{z G^{\prime \prime}(z)}{G^{\prime}(z)}\right)\right\}>0 \quad(z \in \mathbb{U}) .
\end{aligned}
$$

Therefore, by virtue of Lemma $2.5, L(z, t)$ is a subordination chain. We observe from the definition of a subordination chain that

$$
\begin{gathered}
\phi(z)=\frac{\gamma-\beta}{\gamma-\beta+1} G(z)+\frac{1}{\gamma-\beta+1} z G^{\prime}(z)=L(z, 0), \\
L(z, 0) \prec L(z, t) \quad(0 \leq t<\infty) .
\end{gathered}
$$

This implies that

$$
L(\zeta, t) \notin L(\mathbb{U}, 0)=\phi(\mathbb{U}) \quad(\zeta \in \partial \mathbb{U} ; 0 \leq t<\infty) .
$$

Now suppose that $F$ is not subordinate to $G$, then by Lemma 2.3, there exist points $z_{0} \in \mathbb{U}$ and $\zeta_{0} \in \partial \mathbb{U}$ such that

$$
F\left(z_{0}\right)=G\left(\zeta_{0}\right), \quad z_{0} F\left(z_{0}\right)=(1+t) \zeta_{0} G^{\prime}\left(\zeta_{0}\right) \quad(0 \leq t<\infty) .
$$

Hence, we have

$$
\begin{aligned}
L\left(\zeta_{0}, t\right) & =\frac{\gamma-\beta}{\gamma-\beta+1} G\left(\zeta_{0}\right)+\frac{1+t}{\gamma-\beta+1} \zeta_{0} G^{\prime}\left(\zeta_{0}\right) \\
& =\frac{\gamma-\beta}{\gamma-\beta+1} F\left(\zeta_{0}\right)+\frac{1}{\gamma-\beta+1} z_{0} F^{\prime}\left(z_{0}\right) \\
& =z_{0}\left(z_{0} f\left(z_{0}\right)\right)^{\beta} \in \phi(\mathbb{U}),
\end{aligned}
$$

by virtue of the subordination condition (3.3). This contradicts the above observation that $L\left(\zeta_{0}, t\right) \notin \phi(\mathbb{U})$. Therefore, the subordination condition (3.3) must imply the subordination given by (3.19). Considering $F(z)=G(z)$, we see that the function $G(z)$ is the best dominant. This evidently completes the proof of Theorem 3.1.

Remark 3.2. We note that $\delta$ given by (3.2) in Theorem 3.1 satisfies the inequality $0<\delta \leq 1 / 2$.

We next prove a dual problem of Theorem 3.1 in the sense that the subordinations are replaced by superordinations. 
Theorem 3.3. Let $f, g \in \Sigma_{\beta, \gamma}$. Suppose that

$$
\operatorname{Re}\left\{1+\frac{z \phi^{\prime \prime}(z)}{\phi^{\prime}(z)}\right\}>-\delta \quad\left(z \in \mathbb{U} ; \phi(z):=z(z g(z))^{\beta}\right),
$$

where $\delta$ is given by (3.2), $z(z f(z))^{\beta}$ is univalent in $\mathbb{U}$, and $z\left(z I_{\beta, r}(f)(z)\right)^{\beta} \in Q$, where $I_{\beta, \gamma}$ is the integral operator defined by (1.6). Then, the superordination

$$
z(z g(z))^{\beta} \prec z(z f(z))^{\beta}
$$

implies that

$$
z\left(z I_{\beta, \gamma}(g)(z)\right)^{\beta} \prec z\left(z I_{\beta, \gamma}(f)(z)\right)^{\beta} .
$$

Moreover, the function $z\left(z I_{\beta, \gamma}(g)(z)\right)^{\beta}$ is the best subordinant.

Proof. The first part of the proof is similar to that of Theorem 3.1 and so we will use the same notation as in the proof of Theorem 3.1. Now let us define the functions $F$ and $G$, respectively, by (3.5). We first note that by using (3.9) and (3.10), we obtain

$$
\begin{aligned}
\phi(z) & =\frac{\gamma-\beta}{\gamma-\beta+1} G(z)+\frac{1}{\gamma-\beta+1} z G^{\prime}(z) \\
& :=\varphi\left(G^{\prime}(z), z G^{\prime}(z)\right) .
\end{aligned}
$$

After a simple calculation, (3.29) yields the relationship

$$
1+\frac{z \phi^{\prime \prime}(z)}{\phi^{\prime}(z)}=q(z)+\frac{z q^{\prime}(z)}{q(z)+\gamma-\beta^{\prime}}
$$

where the function $q$ is defined by (3.7). Then, by using the same method as in the proof of Theorem 3.1, we can prove that

$$
\operatorname{Re}\{q(z)\}>0 \quad(z \in \mathbb{U}),
$$

that is, $G$ defined by (3.5) is convex(univalent) in $\mathbb{U}$.

Next, we prove that the superordination condition (3.27) implies that

$$
F(z) \prec G(z) \quad(z \in \mathbb{U}) .
$$

Now, consider the function $L(z, t)$ defined by

$$
L(z, t):=\frac{\gamma-\beta}{\gamma-\beta+1} G(z)+\frac{t}{\gamma-\beta+1} z G^{\prime}(z) \quad(z \in \mathbb{U} ; 0 \leq t<\infty) .
$$

Since $G$ is convex and $\operatorname{Re}\{\gamma-\beta\}>0$, we can prove easily that $L(z, t)$ is a subordination chain as in the proof of Theorem 3.1. Therefore, according to Lemma 2.4, we conclude that the superordination condition (3.27) must imply the superordination given by (3.32). Furthermore, since the differential equation (3.29) has the univalent solution $G$, it is the best subordinant of the given differential superordination. Therefore, we complete the proof of Theorem 3.3. 
If we combine Theorems 3.1 and 3.3, then we can obtain the following sandwich-type theorem.

Theorem 3.4. Let $f, g_{k} \in \Sigma_{\beta, \gamma}(k=1,2)$. Suppose that

$$
\operatorname{Re}\left\{1+\frac{z \phi_{k}^{\prime \prime}(z)}{\phi_{k}^{\prime}(z)}\right\}>-\delta \quad\left(z \in \mathbb{U} ; \phi_{k}(z):=z\left(z g_{k}(z)\right)^{\beta} ; k=1,2\right),
$$

where $\delta$ is given by (3.2), $z(z f(z))^{\beta}$ is univalent in $\mathbb{U}$, and $z\left(z I_{\beta, r}(f)(z)\right)^{\beta} \in Q$, where $I_{\beta, r}$ is the integral operator defined by (1.6). Then, the subordination

$$
z\left(z g_{1}(z)\right)^{\beta} \prec z(z f(z))^{\beta} \prec z\left(z g_{2}(z)\right)^{\beta}
$$

implies that

$$
z\left(z I_{\beta, \gamma}\left(g_{1}\right)(z)\right)^{\beta} \prec z\left(z I_{\beta, \gamma}(f)(z)\right)^{\beta} \prec z\left(z I_{\beta, \gamma}\left(g_{2}\right)(z)\right)^{\beta}
$$

Moreover, the functions $z\left(z I_{\beta, \gamma}\left(g_{1}\right)(z)\right)^{\beta}$ and $z\left(z I_{\beta, \gamma}\left(g_{2}\right)(z)\right)^{\beta}$ are the best subordinant and the best dominant, respectively.

The assumption of Theorem 3.4, that the functions $z(z f(z))^{\beta}$ and $z\left(z I_{\beta, \gamma}(f)(z)\right)^{\beta}$ need to be univalent in $\mathbb{U}$, may be replaced by another condition in the following result.

Corollary 3.5. Let $f, g_{k} \in \Sigma_{\beta, \gamma}(k=1,2)$. Suppose that the condition (3.34) is satisfied and

$$
\operatorname{Re}\left\{1+\frac{z \psi^{\prime \prime}(z)}{\psi^{\prime}(z)}\right\}>-\delta \quad\left(z \in \mathbb{U} ; \psi(z):=z(z f(z))^{\beta} ; z f(z) \in Q\right),
$$

where $\delta$ is given by (3.2). Then, the subordination

$$
z\left(z g_{1}(z)\right)^{\beta} \prec z(z f(z))^{\beta} \prec z\left(z g_{2}(z)\right)^{\beta}
$$

implies that

$$
z\left(z I_{\beta, \gamma}\left(g_{1}\right)(z)\right)^{\beta} \prec z\left(z I_{\beta, \gamma}(f)(z)\right)^{\beta} \prec z\left(z I_{\beta, \gamma}\left(g_{2}\right)(z)\right)^{\beta}
$$

where $I_{\beta, r}$ is the integral operator defined by (1.6). Moreover, the functions $z\left(z I_{\beta, \gamma}\left(g_{1}\right)(z)\right)^{\beta}$ and $z\left(z I_{\beta, r}\left(g_{2}\right)(z)\right)^{\beta}$ are the best subordinant and the best dominant, respectively.

Proof. In order to prove Corollary 3.5, we have to show that the condition (3.37) implies the univalence of $\psi(z)$ and

$$
F(z):=z\left(z I_{\beta, r}(f)(z)\right)^{\beta} .
$$

Since $0<\delta \leq 1 / 2$ from Remark 3.2, the condition (3.37) means that $\psi$ is a close-to-convex function in $\mathbb{U}$ (see [19]) and hence $\psi$ is univalent in $\mathbb{U}$. Furthermore, by using the same techniques as in the proof of Theorem 3.4, we can prove the convexity (univalence) of $F$ and so the details may be omitted. Therefore, by applying Theorem 3.4, we obtain Corollary 3.5. 
By setting $\gamma-\beta=1$ in Theorem 3.4, so that $\delta=1 / 2$, we deduce the following consequence of Theorem 3.4.

Corollary 3.6. Let $f, g_{k} \in \Sigma_{\beta, \beta+1}(k=1,2)$. Suppose that

$$
\operatorname{Re}\left\{1+\frac{z \phi_{k}^{\prime \prime}(z)}{\phi_{k}^{\prime}(z)}\right\}>-\frac{1}{2} \quad\left(z \in \mathbb{U} ; \phi_{k}(z):=z\left(z g_{k}(z)\right)^{\beta} ; k=1,2\right)
$$

$z(z f(z))^{\beta}$ is univalent in $\mathbb{U}$, and $z\left(z I_{\beta, \beta+1}(f)(z)\right)^{\beta} \in \mathcal{Q}$, where $I_{\beta, \beta+1}$ is the integral operator defined by (1.6) with $\gamma=\beta+1$. Then, the subordination

$$
z\left(z g_{1}(z)\right)^{\beta} \prec z(z f(z))^{\beta} \prec z\left(z g_{2}(z)\right)^{\beta}
$$

implies that

$$
z\left(z I_{\beta, \beta+1}\left(g_{1}\right)(z)\right)^{\beta} \prec z\left(z I_{\beta, \beta+1}(f)(z)\right)^{\beta} \prec z\left(z I_{\beta, \beta+1}\left(g_{2}\right)(z)\right)^{\beta} .
$$

Moreover, the functions $z\left(z I_{\beta, \beta+1}\left(g_{1}\right)(z)\right)^{\beta}$ and $z\left(z I_{\beta, \beta+1}\left(g_{2}\right)(z)\right)^{\beta}$ are the best subordinant and the best dominant, respectively.

If we take $\gamma-\beta=1+i$ in Theorem 3.4, then we are easily led to the following result.

Corollary 3.7. Let $f, g_{k} \in \Sigma_{\beta, \beta+1+i}(k=1,2)$. Suppose that

$$
\operatorname{Re}\left\{1+\frac{z \phi_{k}^{\prime \prime}(z)}{\phi_{k}^{\prime}(z)}\right\}>-\frac{3-\sqrt{5}}{4} \quad\left(z \in \mathbb{U} ; \phi_{k}(z):=z\left(z g_{k}(z)\right)^{\beta} ; k=1,2\right),
$$

$(z f(z))^{\beta}$ is univalent in $\mathbb{U}$, and $\left(z I_{\beta, \beta+1+i}(f)(z)\right)^{\beta} \in \mathcal{Q}$, where $I_{\beta, \beta+1+i}$ is the integral operator defined by (1.6) with $\gamma=\beta+1+i$. Then, the subordination:

$$
z\left(z g_{1}(z)\right)^{\beta} \prec z(z f(z))^{\beta} \prec z\left(z g_{2}(z)\right)^{\beta}
$$

implies that

$$
z\left(z I_{\beta, \beta+1+i}\left(g_{1}\right)(z)\right)^{\beta} \prec z\left(z I_{\beta, \beta+1+i}(f)(z)\right)^{\beta} \prec z\left(z I_{\beta, \beta+1+i}\left(g_{2}\right)(z)\right)^{\beta} .
$$

Moreover, the functions $z\left(z I_{\beta, \beta+1+i}\left(g_{1}\right)(z)\right)^{\beta}$ and $z\left(z I_{\beta, \beta+1+i}\left(g_{2}\right)(z)\right)^{\beta}$ are the best subordinant and the best dominant, respectively.

\section{Acknowledgments}

The authors would like to express their gratitude to Professor S. Stević for his valuable suggestions. This research was supported by Kyungsung University Grants in 2008. 


\section{References}

[1] S. S. Miller and P. T. Mocanu, Differential Subordinations: Theory and Applications, vol. 225 of Monographs and Textbooks in Pure and Applied Mathematics, Marcel Dekker, New York, NY, USA, 2000.

[2] S. S. Miller and P. T. Mocanu, "Subordinants of differential superordinations," Complex Variables and Elliptic Equations, vol. 48, no. 10, pp. 815-826, 2003.

[3] A. W. Goodman, Univalent Functions. Vol. I, Mariner, Tampa, Fla, USA, 1983.

[4] A. W. Goodman, Univalent Functions. Vol. II, Mariner, Tampa, Fla, USA, 1983.

[5] S. S. Miller and P. T. Mocanu, "Univalent solutions of Briot-Bouquet differential equations," Journal of Differential Equations, vol. 56, no. 3, pp. 297-309, 1985.

[6] S. K. Bajpai, "A note on a class of meromorphic univalent functions," Revue Roumaine de Mathématiques Pures et Appliquées, vol. 22, no. 3, pp. 295-297, 1977.

[7] S. S. Bhoosnurmath and S. R. Swamy, "Certain integrals for classes of univalent meromorphic functions," Ganita, vol. 44, no. 1-2, pp. 19-25, 1993.

[8] A. Dernek, "Certain classes of meromorphic functions," Annales Universitatis Mariae Curie-Skłodowska. Sectio A, vol. 42, pp. 1-8, 1988.

[9] S. P. Dwivedi, G. P. Bhargava, and S. L. Shukla, "On some classes of meromorphic univalent functions," Revue Roumaine de Mathématiques Pures et Appliquées, vol. 25, no. 2, pp. 209-215, 1980.

[10] R. M. Goel and N. S. Sohi, "On a class of meromorphic functions," Glasnik Matematički, vol. 17(37), no. 1, pp. 19-28, 1982.

[11] S. Stević, "The generalized Libera transform on Hardy, Bergman and Bloch spaces on the unit polydisc," Zeitschrift für Analysis und ihre Anwendungen, vol. 22, no. 1, pp. 179-186, 2003.

[12] S. Stević, "A note on the generalized Cesàro operator on Bergman spaces," Indian Journal of Mathematics, vol. 46, no. 1, pp. 129-136, 2004.

[13] S. Stević, "On Libera type transform on the unit disc, polydisc and the unit ball," to appear in Integral Transforms and Special Functions.

[14] S. S. Miller, P. T. Mocanu, and M. O. Reade, "Subordination-preserving integral operators," Transactions of the American Mathematical Society, vol. 283, no. 2, pp. 605-615, 1984.

[15] S. Owa and H. M. Srivastava, "Some subordination theorems involving a certain family of integral operators," Integral Transforms and Special Functions, vol. 15, no. 5, pp. 445-454, 2004.

[16] T. Bulboacă, "A class of superordination-preserving integral operators," Indagationes Mathematicae. New Series, vol. 13, no. 3, pp. 301-311, 2002.

[17] S. S. Miller and P. T. Mocanu, "Differential subordinations and univalent functions," Michigan Mathematical Journal, vol. 28, no. 2, pp. 157-172, 1981.

[18] Ch. Pommerenke, Univalent Functions, Vandenhoeck \& Ruprecht, Göttingen, Germany, 1975.

[19] W. Kaplan, "Close-to-convex schlicht functions," Michigan Mathematical Journal, vol. 1, pp. 169-185, 1952. 


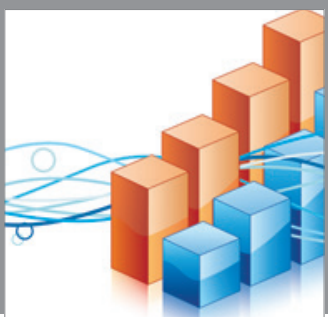

Advances in

Operations Research

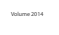

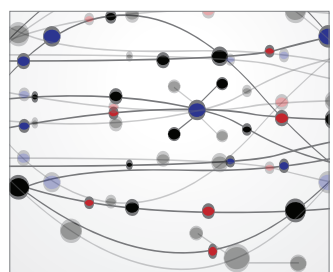

\section{The Scientific} World Journal
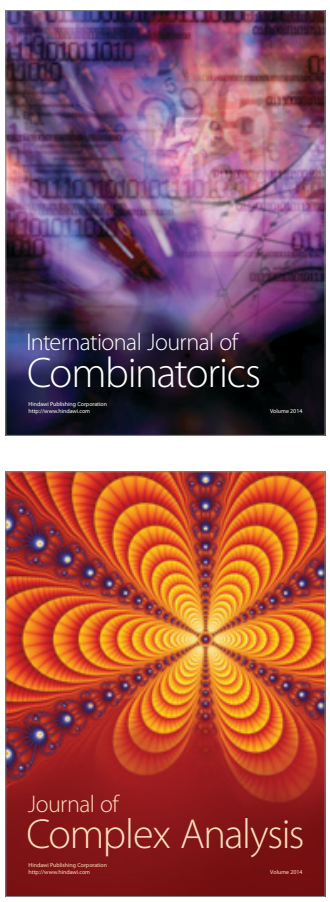

International Journal of

Mathematics and

Mathematical

Sciences
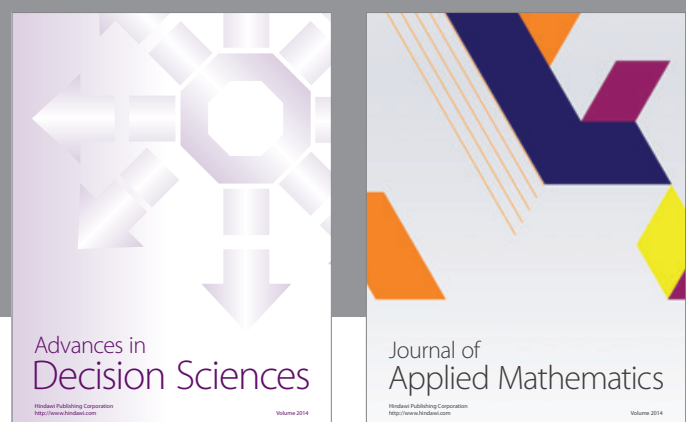

Journal of

Applied Mathematics
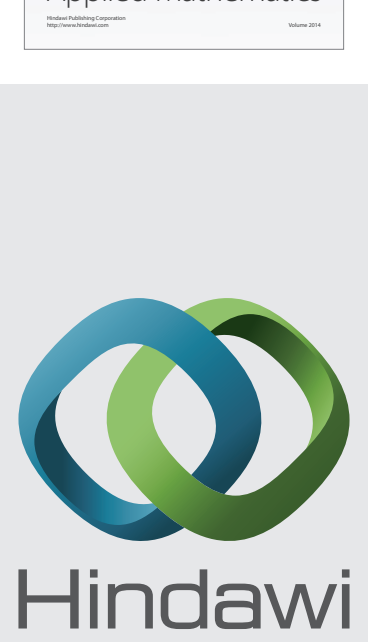

Submit your manuscripts at http://www.hindawi.com
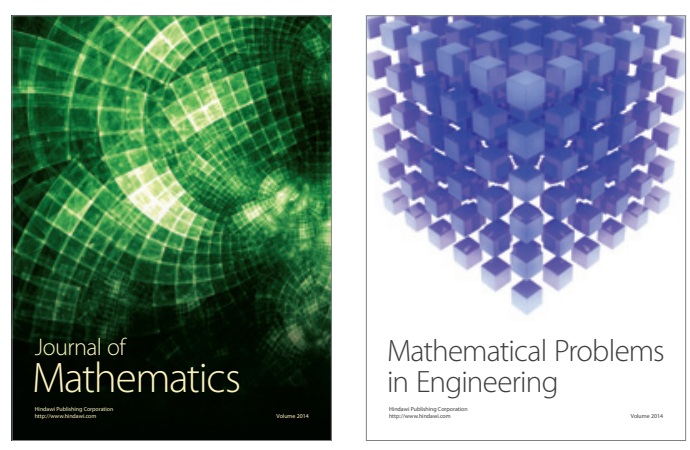

Mathematical Problems in Engineering
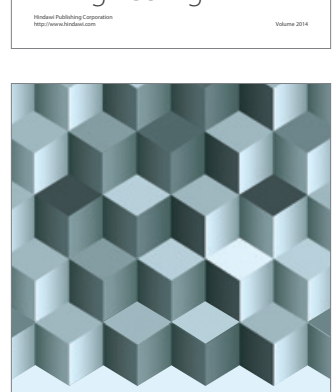

Journal of

Function Spaces
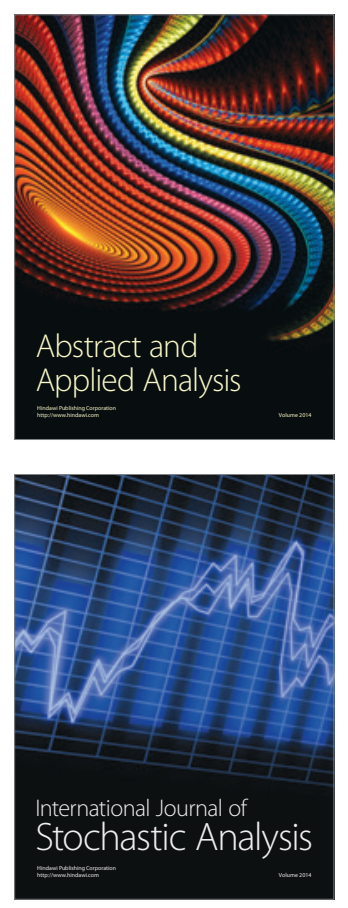

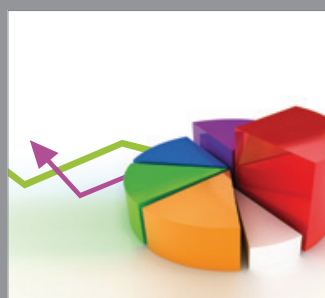

ournal of

Probability and Statistics

Promensencen
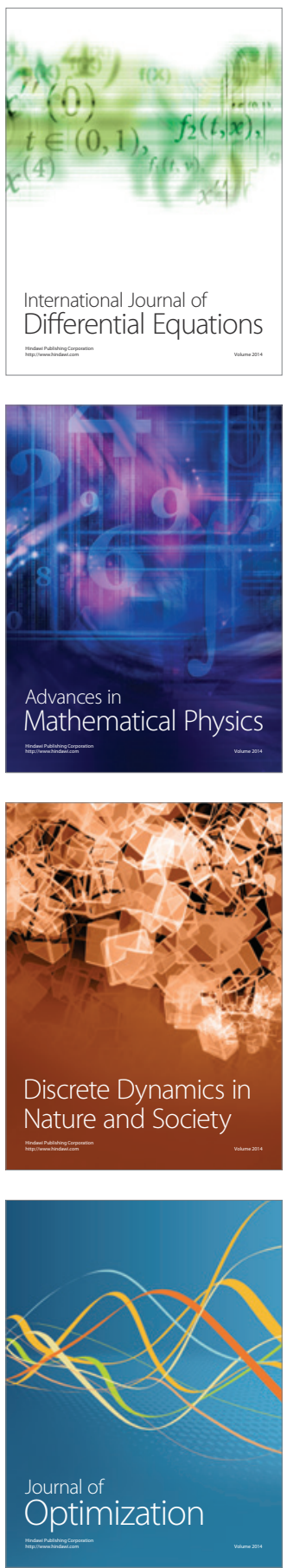